\section{Big Detectors of BGO}

\author{
P. Lecoq, CERN, J.P. Martin, IPN Lyon, \\ M. Schneegans, LAPP Annecy \\ and $M$. Vivargent, CERN, Geneva
}

Particle detection by scintillation counting is a technique that goes back to the beginning of the century when cadmium sulphide screens were used to look at $\alpha$-particles. More transparent scintillators have been introduced since and, particularly over the past 30 years, photodetection methods have been revolutionised. Of all the scintillators, thallium-doped sodium iodide has held pride of place because of its high light output, fine pulse height resolution, rapid response, and, through its high density and high $Z$, good sensitivity. Its main disadvantages are that it is strongly hygroscopic and relatively delicate in the form of a single crystal - necessary for good light transmission. It also suffers irreparable damage after only modest exposure to radiation.

When planning the future experiments for LEP, the Large Electron Positron collider now under construction at CERN, it became urgent to find an alternative scintillator which could be used for big particle calorimeters. One candidate was bismuth germanate $\mathrm{Bi}_{4} \mathrm{Ge}_{3} \mathrm{O}_{12}$ (BGO), crystals of which were first obtained and studied by A. Durif in $1957^{1}$ ) and then grown by the Czokralski method in 1965 by Nietsche ${ }^{2}$ ). In the 1970 's they were produced in small quantities and small sizes mainly as scintillators for nuclear physics and medical applications.

Fig. 2 - Phase diagram of bismuth oxide and germanium oxide.

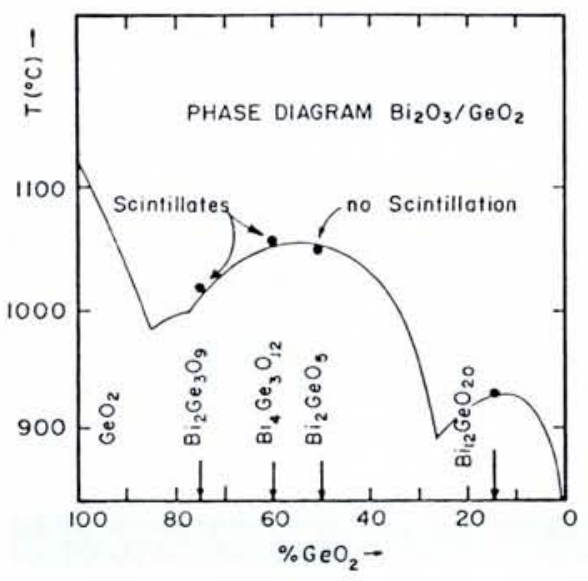

Although BGO emits only about $12 \%$ of the light of $\mathrm{Nal}$ under the same conditions, this is still sufficient for readout by photodiodes (at least in the energy range above a few $\mathrm{MeV}$ ), so it can be used in strong magnetic fields as is frequently necessary in high energy physics. The high concentration of bismuth makes it a very dense material $(7.13$ $\mathrm{g} / \mathrm{cm}^{3}$ ), with a radiation length (the inverse absorption factor) of $1.12 \mathrm{~cm}$, less than half that of $\mathrm{Nal}(2.54 \mathrm{~cm})$. This characteristic is of prime importance for building compact calorimeters with a good spatial resolution. In addition, BGO has a much smaller afterglow than $\mathrm{Nal}$, it is non-hygroscopic, making handling and packing much easier, and is more resistant to radiation damage, which is invaluable for small angle physics around colliders.

The requirements for LEP would, however, be largely in excess of world production and the L3 Collaboration, a European-American-Asiatic collaboration of 36 institutes, decided to invest considerable effort in persuading industry and national laboratories to embark on $R$ \& D programmes with a view to mass producing crystals in large sizes. Their campaign has met with a satisfying response as far afield as China.

\section{BGO Production}

Bismuth oxide $\left(\mathrm{Bi}_{2} \mathrm{O}_{3}\right)$ and germanium oxide $\left(\mathrm{GeO}_{2}\right)$ combine in well defined proportions corresponding to different crystalline states, the phase diagram showing four distinct phases (Fig. 1). At room temperature both $\mathrm{Bi}_{2} \mathrm{Ge}_{3} \mathrm{O}_{9}$ and $\mathrm{Bi}_{4} \mathrm{Ge}_{3} \mathrm{O}_{12}$ (BGO) are strongly luminescent but only BGO monocrystals are suitable for electromagnetic calorimetry. High quality crystals can be grown from either a high purity $(6 \mathrm{~N})$ stoichiometric mixture of $\mathrm{Bi}_{2} \mathrm{O}_{3}$ and $\mathrm{GeO}_{2}$ or, polycrystalline $\mathrm{BGO}$.

The mixture has the following disadvantages:

- a fraction of the bismuth oxide decomposes during the heating and the bismuth reacts with the platinum of the crucible. As a result the platinum needs frequent regeneration;

— a fraction of the bismuth oxide eva-

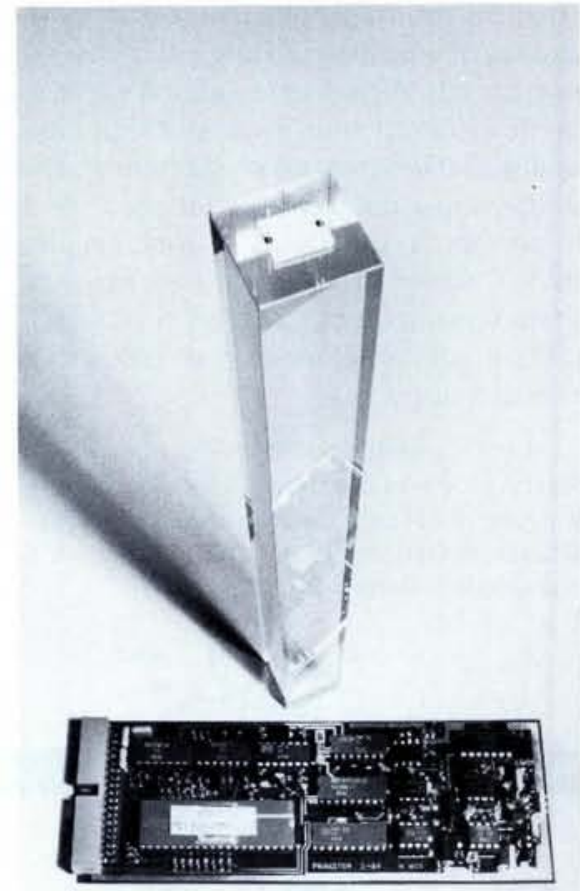

Fig. 1 - A single crystal of BGO, $24 \mathrm{~cm}$ long, with photodiode readout and associated microcomputer.

porates before reacting with the germanium oxide.

These two effects make it difficult to obtain the right stoichiometric composition for the melt, necessary to obtain a high yield and avoid contamination by other crystalline states..

Through cooperation between the L3 Collaboration and industry however, polycrystalline BGO of suitable quality is now produced by sintering. Contamination is less than $1 \mathrm{ppm}$ for any single metal and less than $5 \mathrm{ppm}$ for all metals. The bismuth : germanium ratio is controlled to give a composition to \pm $0.05 \%$ molar $\mathrm{Bi}_{2} \mathrm{O}_{3}$ using a measurement technique sensitive to \pm 0.01 molar \%. The final polycrystalline BGO contains more than $96 \%$ by weight of the $\mathrm{Bi}_{4} \mathrm{Ge}_{3} \mathrm{O}_{12}$ phase. Using this as starting material, big crystals, $3 \times 3 \times 24$ $\mathrm{cm}^{3}$ have been grown.

The normal method of growing is the Czokralski method (Fig. 3 a) starting from a monocrystalline seed in contact with the surface of a polycrystalline BGO melt in a platinum crucible. The seed is fixed at the extremity of a rod rotating at a speed of about $30 \mathrm{rev} / \mathrm{min}$ which is pulled at a speed between 2 and $5 \mathrm{~mm} / \mathrm{h}$ depending on the size of rod required. Rods up to $9 \mathrm{~cm}$ in diameter and $30 \mathrm{~cm}$ in length have been pulled by this technique (Crismatec, Shin-Etsu).

The Bridgeman-Stockbarger method is widely used in China and Japan. Cry- 
Fig. $3-$ Schematic of the three principal methods used industrially for growing single crystals. (a) is the Czokralski method employing a rotating furnace; (b) the Bridgeman-Stockbarger method in which the fusion interface is progressively moved vertically; (c) the heat exchange method where cooling of the melt is produced by an increasing flow of helium. a)

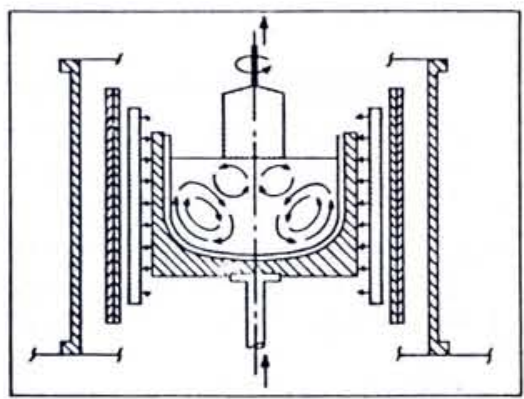

b)

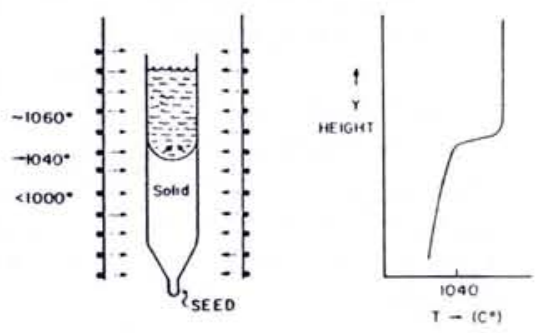

c)

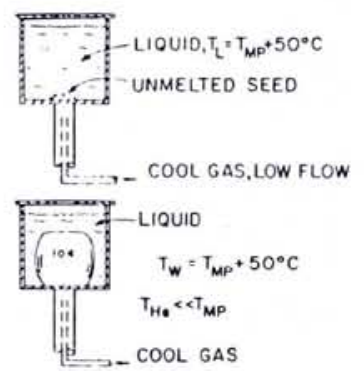

CON GAS
CONERENIRATE
SOLID CRYSTAL
LIOUID MINISCUS stal growth is generated from a seed placed at the bottom of a vertical platinum crucible containing a BGO melt. The solid-liquid interface is moved towards the top of the crucible by displacing vertically the temperature gradient (Fig. 3b). Crystals $3 \times 3 \times 24 \mathrm{~cm}^{3}$ of high quality have also been grown using a similar technique employing horizontal crucibles having the same shape as the crystal (NKK, Institute of Ceramics of Shanghai).

In the floating zone method, crystallisation propagates by displacing a narrow high temperature zone (above the BGO melting point: $1050^{\circ} \mathrm{C}$ ) along a crucible filled with raw material, starting from a seed located at one extremity. This method is at present not widely used by industry.

In addition to these established methods, firms are also now trying to grow big crystals by the heat exchange method (Fig. 3c), where crystal growth propagates from a seed in the lowest part of a platinum crucible raised to a temperature above the melting temperature (about $1100^{\circ} \mathrm{C}$ ). Heat is removed at the level of the seed by a continually increasing helium flow.

The aim of all the methods is to produce at minimum cost which in the first place implies high reliability. Investment in platinum is an important item and even though the majority can be recuperated, reworking used crucibles is expensive. Complex and consequently costly furnaces have to be used in the Czokralski method and the rotating elements introduce technical difficulties. On the other hand, large blocks can be grown which can be cut into several crystals and this reduces the cost per crystal. For the Bridgeman-Stockbarger method, much simpler and less expensive furnaces can be used but the blocks produced are smaller. Separation of the blocks from the crucible has also been a difficult problem, solved by using a crucible made of folded platinum foils.

Today's most promising method however, from the financial point of view, is the heat exchange method, and although so far, only small crystals (up to 5 $\times 5 \times 1.5 \mathrm{~cm}^{3}$ ) have been grown, a vigorous $R \& D$ programme is aimed at improving on this.

\section{BGO Physical and Optical Properties BGO forms an ionic monocrystal: $\left(\mathrm{Bi}^{3+}\right)_{4}\left[\mathrm{GeO}_{4}^{-}\right]_{3}$}

an isomorph of the natural mineral eulitite $\left(\mathrm{Bi}_{4} \mathrm{Si}_{3} \mathrm{O}_{12}\right)$, having interesting optical and electro-optical properties, with a wide range of applications. The scintillation mechanism is complex and not yet completely understood. Recent studies 3.4) favour the model of excitations migrating on bismuth centres by excitonic transfer, which would indicate that it is important to have a very pure material, as impurities can trap the excitation during transfer and dissipate the energy by non-radiative processes.

Owing to a rearrangment of molecular orbitals of emitting centres after excitation, causing dissipation of part of the excitation energy by phonon exchange, the excitation spectrum in the UV range below $300 \mathrm{~nm}$ does not overlap the broad fluorescence band which peaks at $480 \mathrm{~nm}$. Within the emission band however, from $300 \mathrm{~nm}$ to $600 \mathrm{~nm}$, BGO is very transparent and the main problem is the high refractive index (2.15) which makes the coupling between the crystal and the photodiode difficult. Much work is in progress to solve this problem by surface preparation of the crystals to give a specular transmission, using intermediate fixing agents of high refractive index and also developing new windows for the photodiodes.

When exposed to radiation, BGO becomes brown and loses part of its transparency, an effect which saturates at high doses (above 1000 rad). Spontaneous recovery takes place in a few weeks at room temperature (Fig. 4), and in a few hours only at $60^{\circ} \mathrm{C}$. Our under-

\section{UNIVERSITY OF GENEVA}

The Department of Nuclear and Particle Physics has an opening for a position of

RESEARCH ASSOCIATE

to join a group preparing an experiment at LEP. The candidate must have a PhD or equivalent experience in high energy physics. This is a non-permanent position limited to a maximum of six years.

Applications should be sent to:

Prof. E. Herr, Director

Département de physique nucléaire et corpusculaire 24, quai Ernest-Ansermet, CH - 1211 Geneva 4 


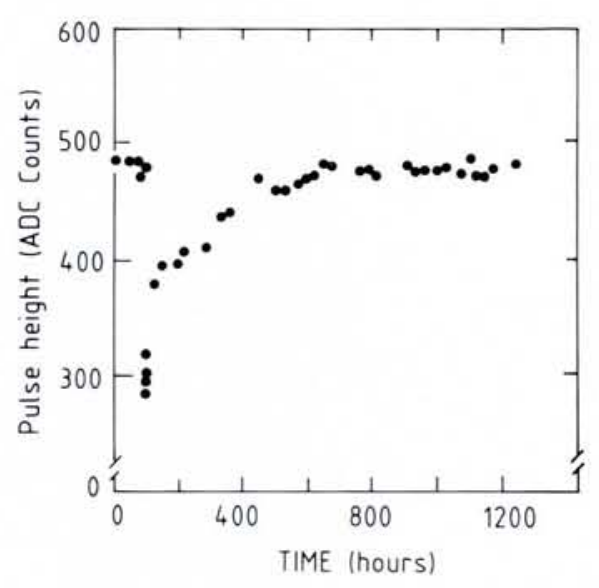

Fig. 4 - Recovery of BGO sensitivity following a sudden massive dose of radiation resulting from a beam loss.

standing of the radiation damage process is the excitation of metastable states related to impurities in the crystal. One sees here again the importance of controlling the purity level of the raw material and the growing process to obtain good quality crystals.

\section{Applications of BGO \\ Nuclear medicine}

Positron emission tomography is now extensively used because it seems to be the best high resolution technique for the regional measurement of tracer compounds after injection into the human body, mainly the heart and the brain. For a long time, resolution was limited because NAI (TI) scintillators were unable to stop the annihilation $\gamma$ ray of $511 \mathrm{keV}$ in a sufficiently short distance to provide the desired localization. BGO detectors are more efficient in small sizes giving better spatial resolution; they are therefore preferred for high resolution tomography in systems where no attempt is made to utilize the time of flight information. Another advantage of $B G O$ is the low afterglow which means that intense fluxes of $\gamma$ rays can be employed.

\section{Industrial applications:}

In oil well logging, fast in-situ analyses are needed of the geological formations through which the drill has passed to determine if hydrocarbons are present, in what amount and if they can be extracted from the rock. The size of the detectors used are limited to the reduced diameter of the borehole, generally about $8 \mathrm{~cm}$. The large stopping power of $B G O$ is an advantage because it allows a better miniaturization of the detector. Moreover, the non-hygroscopic nature of the $B G O$ reduces the demands on packaging. BGO however experiences a sharp decrease in light output above room temperature, so that cooling has to be provided.

Scanners are used by nuclear fuel manufacturers throughout the world to inspect fuel rods and detect deviations from specifications. BGO allows an improvement of the spatial resolution of these scanners so that a more detailed study of the fuel rods can be made.

\section{Fundamental research:}

Because of their compactness, BGO detectors can be installed on satellites to provide a better evaluation of the electron flux in space for a given energy range, or used in space shuttle experiments to make investigations of the $\gamma$ ray background induced in the orbiter by exposure to the radiation belts, or to undertake $\mathrm{X}$ - and $\gamma$-ray spectroscopy of the sky.

Many studies in nuclear physics require an accurate spectroscopic analysis. Germanium semiconductor detectors are widely employed, but an important background in the measured $\gamma$-ray spectra arises from Compton scattering in the detector and the escape of the scattered gamma. For such events, only a fraction of the gamma energy is measured, but the loss can be compensated by using a scintillation detector around the semiconductors to measure the radiation that escapes. BGO is then very interesting because of its high stopping power. This is also valuable in stopping fast charged particles coming from heavy ion collisions, allowing a measurement of their total energy. Associated with other detectors that do not stop these fast particles, but measure the energy loss on passing through, we can form an $E / \triangle E$ telescope able to identify protons in the few hundred $\mathrm{MeV}$ range.

\section{High Energy Calorimetry}

The very good resolution obtained with BGO when measuring the energy of electromagnetic showers, and its short radiation length, make it particularly suitable for high energy precision calorimetry. It is used or proposed in several experiments with limited solid angle coverage.

At LEP, where the electron and photon energies may reach $100 \mathrm{GeV}$, a depth of BGO of more than $20 \mathrm{~cm}$ will be necessary, with a full solid angle coverage around the $\mathrm{e}^{+} \mathrm{e}^{-}$interaction region. In the $\mathrm{L} 3$ experiment, one aspect of which is a very precise reconstruction of the muon momenta, compact calorimeters surrounding a small central track chamber are vital. For these a total BGO volume of $1.6 \mathrm{~m}^{3}$ in crystals of about 150 $\mathrm{cm}^{3}$ is foreseen.

In 1982, a stack of nine BGO crystals was tested by members of L 3 in a CERN beam up to $19 \mathrm{GeV}$ and its good behaviour confirmed. In 1983, a matrix of 25 parallelepiped crystals was extensively studied in the $X 3$ beam at the CERN SPS between 1 and $50 \mathrm{GeV}^{5}$ ). Good linearity over the whole energy range and resolutions near $1 \%(\triangle E / E)$ were observed from 4 to $50 \mathrm{GeV}$ with $24 \mathrm{~cm}$ long crystals. At lower energies, the contributions of electronic noise and of beam momentum spread have so far prevented the ultimate $B G O$ resolution being reached.

A position resolution of the shower centre of gravity of $1-3 \mathrm{~mm}(\sigma)$ has been achieved and a hadron contamination of less than 0.002 measured between 2 and $10 \mathrm{GeV}$, using only the energy-momentum comparison.

This year, a matrix of 25 tapered crystals, $24 \mathrm{~cm}$ long, of a shape very near that of the L 3 calorimeter crystals, i.e. a truncated pyramid with square bases of $2 \times 2$ and $3 \times 3 \mathrm{~cm}^{2}$, is being studied in the $X 3$ beam. Preliminary results show a behaviour very similar to the parallelepiped crystals once the light collection has been made uniform along the crystal, and energy resolutions near 1\% have again been measured.

In parallel with this work, extensive studies are going on in the laboratories of the L 3 Collaboration in order to reduce the electronic noise from the photodiodes, and increase the amount of light which can be extracted from a crystal, to optimize the surface treatment of the tapered crystals for uniformity of light collection, and to find the characteristics of a crystal resistant to radiation. Moreover, methods for energy calibrating the crystals of the final L 3 calorimeter, are being thoroughly investigated using sources, Van de Graafs, betatrons and physics reactions. It is planned to position each crystal in an electron beam at various energies, before lowering the calorimeter into the LEP pit. Also the possibility of monitoring each crystal with fibre distributed light from lasers, LED generators or Xe lamps is being studied.

\section{REFERENCES}

1. Durif A., C.r. hebd. seanc., Paris 244 (1957) 2815.

2. Nietsche R., J. Appl. Physics, 36 (1965) 2358.

3. Moncorge R. et al. Temperature Dependent Luminescence of $\mathrm{Bi}_{4} \mathrm{Ge}_{3} \mathrm{O}_{12}$. Discussion on possible models. J. of Luminescence 14 (1976) 337-348.

4. Boulon G., Pedrini D. et al. Private communication.

5. Bakken J. et al. CERN Preprint and NIM paper. 